\title{
Measuring and Testing Area Navigation Procedures with GNSS
}

\author{
Andrej Novák $^{1, *}$, Filip Škultéty $^{1}$, Branislav Kandera, and Tomasz Łusiak ${ }^{2}$ \\ ${ }^{1}$ Žilinská univerzita v Žiline, Air Transport Department, Univerzitna 8215/1 01026 Zilina, Slovakia \\ ${ }^{2}$ Lublin University of Technology, Department of Thermodynamics, Fluid Mechanics and Aviation \\ Propulsion Systems, Nadbystrzycka 36, 20-618 Lublin, Poland
}

\begin{abstract}
This paper deals with the flight testing and measuring of the GNSS (RNAV) with flying laboratory - AeroLab 1 at the University of Žilina. The paper focusses on the comparative aerial measurements of the GNSS RNP APCH which is approved and published at the Žilina airport. The GNSS procedure was put into operation in May 2017 and is subjected to regular checks. Results of flight checking must be within the limits of the prescribed tolerances defined in the ICAO Annex 10. Our practical measurements were divided into two flights. The displayed outputs on the console were compared with the reference values and subsequently evaluated in the conclusion in this paper. Our practical comparative measurements show results that comply with ICAO standards and thus the tested RNP APCH is functional and can be used in mountains and river areas.
\end{abstract}

\section{Introduction to problems solving}

The WAAS provides augmentation, including integrity broadcasts, differential corrections, and additional ranging signals to the standard GPS signal. It provides the accuracy, integrity, availability, and continuity required to support oceanic, remote-area, en route, terminal, non-precision approach, and APV approach phases of flight.

WAAS utilizes a network of wide-area reference stations (WRS) that receive and monitor the GPS signals. Data from these reference stations are transmitted to one of two wide-area master stations (WMS), where the validity of the signals from each satellite is assessed and wide-area corrections are computed. These validity (integrity) messages and wide-area corrections are transmitted to aircraft via Geostationary Earth Orbit (GEO) communications satellites that serve as additional sources of GPS ranging signals, increasing the number of satellites available to the system users. The WAAS signal is transmitted on the same frequency (L1 - 1,575.42 MHz) and with the same type of codedivision multiplex modulation as the GPS Standard Positioning Service (SPS) signal. This allows a WAAS receiver to acquire and process both the GPS and WAAS broadcasts. An integrity message transmitted by the WAAS provides the user with a direct verification of the integrity of the signal from each satellite in view.

* Corresponding author: Andrej.Novak@fpedas.uniza.sk 
Ground Based Augmentation System (GBAS) is a safety-critical system consisting of the hardware and software that augments the Global Positioning System (GPS) Standard Positioning Service (SPS) to provide precision approach and landing capability. The positioning service provided by GPS is insufficient to meet the integrity, continuity, accuracy, and availability demands of precision approach and landing navigation. GBAS provides a precision approach service and a positioning service, which provides horizontal position information to support RNAV operations in the terminal area. GBAS augments the GPS SPS in order to meet these requirements. These augmentations are based on differential GPS concepts. GBAS refers to any system compliant with the existing ICAO standards [1].

GBAS is comprised of ground equipment and avionics. The ground equipment includes four or five reference receivers and associated antennas, an electronics cabinet, and a very high frequency (VHF) data broadcast (VDB) transmitter and associated antenna. The ground equipment is complemented by GBAS avionics installed on the aircraft.

The VDB broadcasts the GBAS signal throughout the GBAS coverage area to avionics in GBAS-equipped aircraft. The GBAS reference receivers independently measure GPS satellite pseudo-range and carrier phase and generate differential carrier-smoothed-code corrections that are eventually broadcast to the user via a $31.5 \mathrm{kbps}$ VHF data broadcast (in the $108-118 \mathrm{MHz}$ band) that also includes safety and approach geometry information. Aircraft landing at a GBAS-equipped airport will be able to perform precision approach operations to at least Category I weather minima. The GBAS approach charts are titled "GLS RWY xx" (GBAS Landing System) [2].

The navigation guidance on terminal segments for the aircraft to transition into a GBAS precision approach final or missed approach may be provided by either RNAV or groundbased systems. Use of the appropriate chapter(s) in this order is required for flight inspection of procedural segments for obstructions, procedural design, navigation guidance, and RNAV coding. This chapter provides inspection guidance and tolerances for any system meeting ICAO GBAS minimum standards. The GNSS is divided to three groups:

- Global Navigation Satellite System (GNSS), such as:

- GPS

- GLONASS

- GALILEO

- Space-Based Augmentation System (SBAS), such as:

- Wide Area Augmentation System (WAAS)

- European Geostationary Navigation Overlay Service (EGNOS)

- GPS Aided Geo Augmented Navigation system (GAGAN)

- MTSAT Satellite Augmentation System (MSAS)

- System for Differential Corrections and Monitoring (SDCM)

- Ground-Based Augmentation Systems (GBAS), such as:

- Local Area Augmentation System (LAAS)

Similarly, Area Navigation (RNAV) is a method of navigation which permits aircraft operation on any desired flight path within the coverage of ground- or space-based navigation aids or within the limits of the capability of self-contained aids, or a combination of these. RNAV includes performance-based navigation as well as other operations that do not meet the definition of performance-based navigation. Flight Management Systems (FMS) with multiple sensors and Global Positioning System (GPS) navigators are most common. These systems navigate with reference to geographic positions called waypoints [3]. Multi-sensor RNAV equipment determines aircraft position by processing data from various input sensors. Unlike early RNAV systems which used only Very high frequency Omnidirectional Range (VOR)/ Distance Measuring Equipment (DME) Rho-Theta for position fixing, multi-sensor navigation systems employ a variety of sensors. 
These various sensors may be used by the navigation computer individually, or combined in various ways (based on internal software programming) to derive aircraft position. Navigation values, such as distance and bearing to a waypoint (WP), are computed from the derived latitude/ longitude to the coordinates of the waypoint. Course guidance is generally provided as a linear deviation from the desired track of a great circle route. RNAV (GNSS) procedures consist of sequenced ARINC 424 coded path and terminators and waypoints. The desired course may be pilot selectable (e.g., pseudo course or go direct) or may be determined by the navigation database, based on the ground track coded between successive waypoints. Use of a combination of different ARINC 424 leg path and terminators provides the desired ground track and vertical path of a flight procedure [4].

Many RNAV procedures include vertical guidance (VNAV). Vertical guidance is provided as a linear deviation from the desired vertical path, defined by a line joining two waypoints with specified altitudes, or as a vertical angle from a specified waypoint. Computed positive vertical guidance is based on barometric, satellite elevation, or other approved systems. The desired vertical path may be pilot selectable, or may be determined by the VNAV computer, with computations based on the ARINC 424 coding in the navigation base. RNAV approaches with vertical guidance are classified as Approach with Vertical Guidance (APV) [5,6].

According to our literature review we analyzed the paper Non directional beacons checking (Pitor, J., 2014). And other research papers such as Airborne Weather Surveillance Radars for Increasing Air Transport Safety (Labun, J., 2017), and the paper Determining acceptable level of safety of approach to landing (Kraus, J.,2016), which are similar to our research [7].

\section{Methodology}

The requirements navigation performance and aircraft performance is defined according ICAO document for Flight Inspection Manual (ICAO, DOC 8071) [8]. But we able generally set that aircraft avionics configuration must be appropriate to support the procedure to be flight inspected. Flight Inspection of RNAV Standard Instrument Departure (SID(s)), airways, and Standard Terminal Arrival Route (STAR(s)) may be accomplished with any flight inspection aircraft capable of the procedure's ARINC 424 path and terminators. RNAV approach charts provide separate minima for Lateral Navigation (LNAV), Lateral and Vertical Navigation (LNAV/ VNAV), LP, and LPV. Inspection of an RNAV procedure with vertical guidance requires an appropriately equipped flight inspection aircraft. The navigation database must be verified to a current navigation database is installed. Use waypoint data from the FMS/ GPS navigation database when available. When official government source ARINC 424 navigation data is available, it must be used for the flight inspection.

The evaluation of procedure data must be prior to the procedure being flown, the navigation database ARINC 424 path and terminator data accuracy must be evaluated by comparison to the official source procedural path or terminator data. This data is found on official ICAO or EASA documents. The navigation database path and terminator coding must match the official source procedure coding documents. The ARINC 424 data evaluation may be completed by a desk-top method or by utilizing an RNAV (GNSS) system on board the flight validation aircraft. (ARINC 424 is an aeronautical specification developed and maintained by the industry which has been used for the exchange of navigation and communication data between commercial data suppliers and avionic system manufactures.) [9]. The desk-top data evaluation is the preferred method. A desk-top method involves a physical comparison, in combination with a computer software tool with 
the capability of displaying the navigation database path and terminator coding and other pertinent coding data in a detailed format, which permits the ARINC 424 coding to be verified against the official source documents. The desktop data evaluation should be detailed enough to include the following ARINC 424 elements for evaluation:

- Procedure Name, Runway

- Transition

- Fix/Waypoint Name

- Distance of leg

- Sequence Number

- Altitude

- Use (For Example: IAF,

- Latitude

FACF, FAF)

- Longitude

- Leg Type (For Example: IF, $\mathrm{TF}, \mathrm{CF})$

- $\operatorname{Turn}(\mathrm{R}, \mathrm{L})$

- Fly-Over (FO) /Fly-By (FB)

- Mag Course or True Course,

- Threshold Crossing Height

- Datum Check

- Vertical Angle

- FAS Data Block Check

- CRC Remainder Check RNAV Track

When approved desktop software and documenting process is used to validate the ARINC 424 coding, some segments of a procedure may not have to be flown. In all cases, the final and missed approach segments of an approach procedure must be flown in an aircraft and checked for flyability and obstructions. In addition, at least two miles of the intermediate segment prior to the FAF must be flown for proper avionics activation of an approach. An approved desktop process for validating ARINC 424 coding satisfies all the requirements to check and validate the ARINC 424 coding without actually flying it in an aircraft. However, there are other conditions and requirements that must be considered before eliminating a segment of an IFP from the in-flight check.

\subsection{Obstacle clearance, communications, and radar coverage}

Obstacle Clearance may be considered satisfactory for procedure segments at or above previously established Minimum IFR Altitudes as described in ANNEX DOC 8071 and DOC 8168 [10].

Communications may be considered satisfactory for procedure segments when there are existing procedures in the same area that require communication with the servicing Air Traffic control ATC). The controlling ATC facility may be contacted directly to confirm communication coverage at the specific locations and altitudes.

Radar coverage may be considered satisfactory for procedure segments when the controlling ATC facility confirms there is radar coverage in the area concerned at or below the required altitude. However, any radar fix identification requirements must be checked in accordance with procedures described in ANNEX DOC 8071.

A procedure segment may still require a flyability check during Flight Validation. Follow the steps in the checklist below for determining which additional segments must be checked for flyability in an aircraft.

When using aircraft avionics to validate ARINC 424 coding, an appropriately equipped aircraft would include a suitable RNAV (GNSS) system capable of displaying and identifying, to the flight inspector, each type of ARINC 424 path and terminator of the flight procedure. Aircraft RNAV (GNSS) systems contain their own magnetic variation data, which may not be current. When using an aircraft RNAV (GNSS) system, verify that true course to next waypoint, distances, and the Flight Path Angle (FPA) indicated on the RNAV (GNSS) system accurately reflects the procedure design. When evaluating RNAV course legs like CF, FC, CA, CI, FA, FC, FM and holding legs (HM, HF, HA), compare 
aircraft navigation performance with the instrument procedure design. Do not apply any tolerance to course-to-fix values. Out-of-tolerance values must be resolved with the procedure designer.

The navigation system status determines the status of the required navigation system(s) (e.g., DME, GPS, GBAS, SBAS) before every flight inspection and after an inspection that detects anomalies. NOTAM(s) and GPS Service Interruptions (interference testing) location and schedule should be considered.

\section{Technical Specification of Flight Laboratory and Practical Measuring}

The Flight Laboratory consists of: technology carrier Piper Seneca V, PA-34-220T, Airfield technology laboratory console, AT 940, Data processing applications: WinFIS, V. 10.14, SPU Version 6.3r1 [11].

The measuring console consists of two segments:

- On-board segment,

- Ground segment.

\subsection{On-board segment}

The on-board segment consists of antenna system, ASU (Avionics Sensor Unit), SPU (Signal Processor Unit) and external avionics sensors. The system is powered by a 28V DC on-board network, and it is separated from the on-board power supply network. The system includes AHRS (Attitude and Heading Reference System) and magnetic compass.

\subsubsection{Avionics Sensor Unit (ASU)}

The Avionics Sensor Unit (ASU) contains a Multi-Mode Receiver (MMR) that receives the signals from the navigation aids being inspected. The MMR combines the ILS, VOR, MKR, DME and ADF functions into a single unit, thereby reducing size, weight, and power requirements. The signals required for flight inspection are output from the avionics sensors, conditioned by circuit boards in the ASU, and output to the Signal Processing Unit (SPU). In the standard AT-940 ASU a dual-function GPS/VHF unit is installed which includes a GPS Technical Standard Order (TSO) receiver and VHF communications transceiver. The GPS (TSO) receiver provides the hardware required for inspection of GPS/GNSS procedures in accordance with ICAO recommendations. The VHF communications transceiver allows the FIS operator to communicate with navigation aid ground engineers during flight inspections.

NAV and GS Receiver Outputs. The MMR contains ILS and VOR receivers, which receive the radio signals from the facility being inspected. The detected LLZ/VOR and Glide Path composite signals are output from the receivers conditioned, and processed by the SPU to measure ILS deviations and modulations, VOR bearing, VOR modulation percentages and FM deviation required for flight inspection and calibration. The automatic gain control (AGC) voltages from the receivers are output to the SPU for measuring the RSL (Received Signal Level) inputs to the receivers [12].

\subsection{Ground segment}

The ground segment consists of a ground reference station with a ground-to-aircraft data communication module operating at a frequency of $868 \mathrm{MHz}$. The ground reference station 
includes a reference antenna with a mast, an omni-directional antenna data link, a reference GPS and GLONAS receiver and a power supply (battery).

\subsection{Data processing application software WinFIS}

WinFIS is the data recording and analysis program used with the AT-940/AT-950 flight inspection system. WinFIS runs under Microsoft Windows and takes full advantage of the Windows graphical user interface and multitasking capabilities. Using WinFIS the FIS operator can view, analyze, and print recordings while a new recording is being made in the background.

\section{Practical measuring on en-route and approach LZZI}

During the partial measuring we testing the GNSS procedure for Standard Arrival (STAR) and approach procedure (APCH) for airport Žilina (LZZI) (See Fig. 1 and Fig. 2). During this test we use the testing procedure according the ICAO manual DOC 8071 and FAA 8200.1D (UNITED STATES STANDARD FLIGHT INSPECTION MANUAL). On the onboard flight laboratory, we use flight inspection system with WinFis, which is used for recording and post analyzing measured signal. For our measuring are important:

- Cross Track Error,

- Along Track Error,

- Flight Technical Error.

Cross Track Error (XTRK): This trace represents the cross track error between the sensor receiver and the reference receiver. The base line for the cross track error trace is the center of the graph and each line represents an error of 200 meters. Left error will displace the trace above the base line and right error will displace the trace below the base line. Default color is blue.

Along Track Error (ATRK): This trace represents the along track error between the sensor receiver and the reference receiver. The base line for the along track error trace is the center of the graph and each line represents an error of 200 meters. Left error will displace the trace above the base line and right error will displace the trace below the base line. Default color is dark green.

Flight Technical Error (FTE): This trace represents the horizontal displacement of the aircraft from the nominal flight path. The base line for the flight technical error trace is the center of the graph and each line represents an error of 200 meters. Left error will displace the trace above the base line and right error will displace the trace below the base line. Default color is light green.

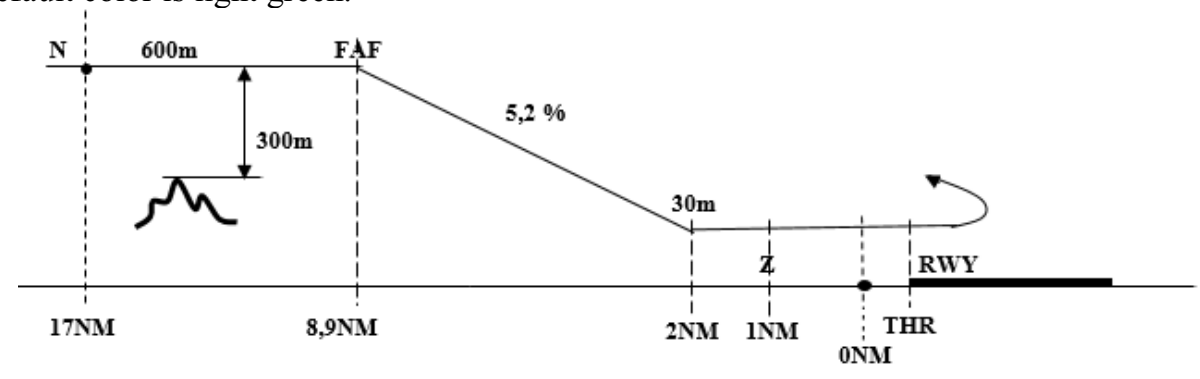

Fig. 1. Measurement Procedure - Vertical Profile. Source: authors 


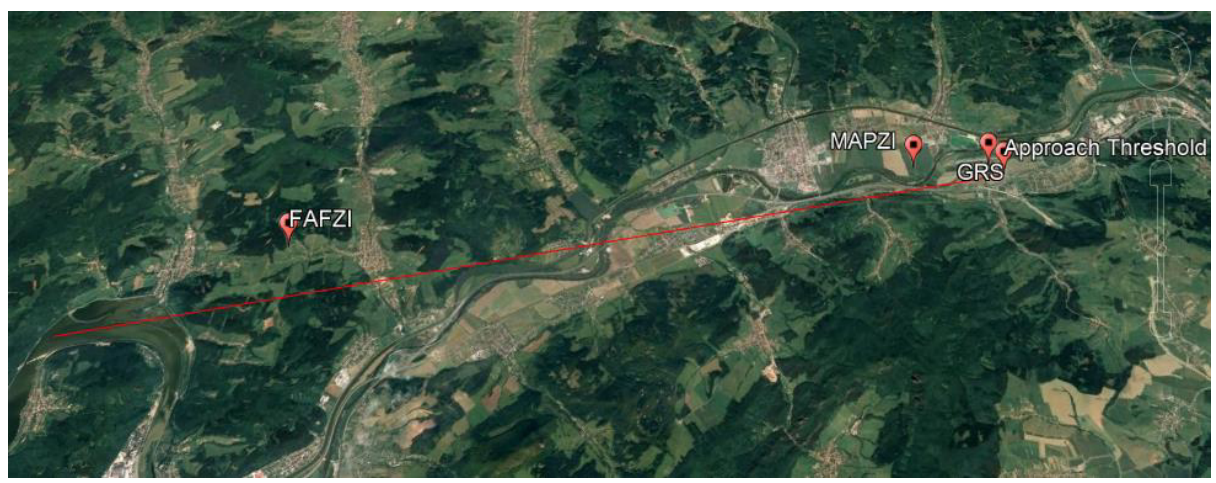

Fig. 2. The Maps of measuring scenario for Airport Zilina. Source: authors

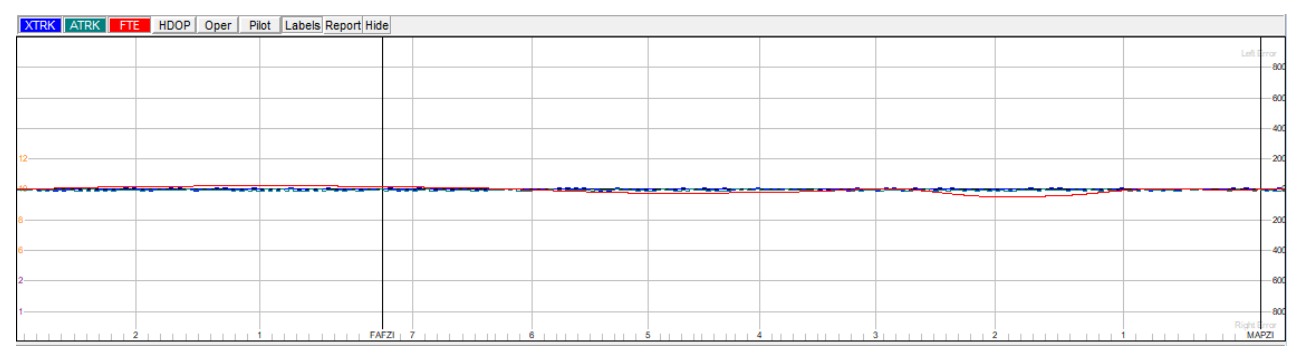

Fig. 3. The Measuring output from measuring scenario for Airport Zilina on Flaying Laboratory. Source: authors

The Figure 3. graphically depicts recorded measurement values for RNP APCH/LZZI, recorded max value: XTRK $0.3 \mathrm{~m}$ ATRK $0.1 \mathrm{~m}$, FTE $1.2 \mathrm{~m}$ (Note: Measurements were carried out for distances of up to $10 \mathrm{NM}$, no measurements were done for a maximum distance of $25 \mathrm{NM}$ ).

The other requirements for the measuring are more subjective, this means for the pilot and system operator visual check, for example obstacle check, runway lights visual system and etc. The measured parameters Cross Track Error and Along Track Error is according to expected value. Flight Technical Error measured parameters, corresponding with real horizontal displacement of the aircraft from the nominal flight path and the max. measured value is relevant to the real situation during measuring day.

\section{Conclusion}

Flight measurement is performed for each aeronautical space equipment during which time regular checks and measurements of the specified parameters are carried out. If these measurements are performed on the equipment or in its close vicinity and the results are within the prescribed tolerances defined in part of Annex 10, then it can be assumed that the signal emitted by this equipment will meet the prescribed requirements at the point of assumed occurrence of the aircraft as well. In addition, the signal of multiple devices is formed into the resulting form only at distances that are beyond the reach of ground measuring and control equipment.

Our measurements have confirmed the fact that the GNSS device is transmitting a signal in accordance with Annex 10, and the signal ripple is within the tolerance. According this measurement Cross Track Error, Along Track Error and Flight Technical Error which is measured in meter is in tolerance. 
Acknowledgements: This paper is published as one of the scientific outputs of the project: „New technologies and best practices in education in the Air Transport and Professional Pilots", KEGA 011ŽU-4/2018 and using testing laboratory from project: „Centre of Excellence for Air Transport ITMS 26220120065“.

\section{References}

1. EUROCONTROL, ARINC $\quad 424 \quad$ Specification. Source:http://www.eurocontrol.int/articles/arinc424-specification (2018)

2. European GNSS Agency: EGNOS Safety of Life (SoL) Service Definition Document; Source: http:// www.egnos-portal.eu/sites/default/files/Brochure-SoL_web.pdf (2015)

3. Overview of MSAS MTSAT Satellite-based Augmentation System For ICG-3; Office of Aeronautical Satellite Systems, ATS Engineering Division, Japan Civil Aviation Bureau; Source: http:// www.oosa.unvienna.org/pdf/sap/2003/vienna2/presentations/08am/tanabe.pdf (2003)

4. N. Neelakantan, Overview of Indian Satellite Navigation Programme; Source: http:// www.unoosa.org/pdf/icg/2010/ICG5/18october/05.pdf (2014)

5. ICAO: Status update of GNSS activities in India, The Sixth Meeting of Ionospheric Studies Task Force (ISTF/6), Bangkok, Thailand, 19 - 21 January 2016; Source: http:// www.icao.int/APAC/Meetings/2016\%20ISTF6/IP05_IND\%20AI.3\%20\%20Status $\% 20$ update $\% 20$ of $\% 20$ GNSS\%20activities.pdf (2016)

6. ANNEX 10,VOLUME 1 - AERONAUTICAL TELECOMMUNICATIONS - RADIO NAVIGATION AIDS, ICAO 2015 (2015)

7. DOC 8071, Volume 1 - MANUAL ON TESTING OF RADIO NAVIGATION AIDS, VOLUME 1 - TESTING OF GROUND-BASED RADIO NAVIGATION SYSTEMS, ICAO, Fourth Edition - 2000

8. J. Pitor, F. Skultety, K. Götz. Transport and communications: scientific journal. 2, pp. 12-16 (2014)

9. J. Labun, P. Kurdel, M. Češkovič, A.N. Sedláčková,Transportation Research Procedia, pp. 156 (2017)

10. J. Kraus, Transport Means - Proceedings of the International Conference 2016 (2016)

11. FAA, United States Standard Flight Inspection Manual, 8200.1D, Departments of the Army, the Navy, and the Air Force and the Federal Aviation Administration (2015)

12. A. Novák, K. Havel, M. Janovec, Transportation Research Procedia, pp. 117 (2017) 